\title{
Deep decarbonization faces deep challenges
}

\author{
By Prachi Patel \\ Feature Editor Vickie Gunderson
}

$\mathrm{I}$ n December 2015, delegates from 195 nations met in Paris to sign a momentous agreement to voluntarily curb their countries' carbon emissions. These countries now face the immense challenge of keeping the planet's temperature from rising more than $2^{\circ} \mathrm{C}$ to avoid the worst effects of climate change.

Meeting that goal is a tall order. It requires deep cuts to carbon emissions from every major sector of the world's economy: power, agriculture, industry, transportation, and infrastructure.

The power sector will underpin these deep decarbonizing efforts. For one, it's one of the biggest carbon culprits: Burning fossil fuels for electricity and heat accounts for a quarter of the world's greenhouse gas emissions. The power sector is also easier to target. "There's the opportunity to cut emissions more rapidly than other sectors because of the various cost-effective options available, from low-carbon substitutes for fossil fuels to energy efficiency," said Jesse Jenkins, a researcher at the MIT Energy Initiative. Plus, decarbonizing the power sector will have a vast ripple effect, "if we use clean electricity to provide heat, run cars, and power industry."

Current voluntary pledges toward Paris goals will result in the world warming by around $3^{\circ} \mathrm{C}$, an analysis in Nature Climate Change showed last year. Keeping warming below $2{ }^{\circ} \mathrm{C}$ will require driving down the power sector's emissions by at least $80 \%$, ideally to zero, and maybe even negative, in which carbon dioxide is extracted from the atmosphere.

The challenge is to decrease power sector emissions at minimal cost while uplifting standards of living and electrifying the 1.1 billion, or $15 \%$ of the global population without access. New materials and technologies could help bring down costs. But the biggest barrier to decarbonizing electricity isn't just technical, it is political will and financial support. Meeting Paris Agreement goals will require, "significant policy reforms, aggressive carbon pricing, and additional technological innovation," according to the International Renewable Energy Agency (IRENA).

There is no one-size-fits-all solution for decarbonizing electricity. Countries'strategies depend on their development level, current carbon emissions, and regionally available resources. For some, it involves replacing fossil fuels with renewables such as wind, solar, and geothermal. Others are choosing nuclear power, or more advanced technologies such as carbon capture. But for each country, meeting their goal will depend on if technology, social, and political drivers can effectively stimulate the private sector.
For example, the 16 members of the Deep Decarbonization Pathways Project (DDPP), a global collaboration of research teams, are split into four groups: low emissions per capita and moderate income (Brazil, Indonesia, India, Mexico); medium emissions and moderate income (China, Russia, South Africa); medium emissions and high income (France, Germany, Italy, Japan, Korea, UK); and high emissions and high income (Australia, Canada, US). These 16 countries emit around $70 \%$ of the world's carbon.

India is on track to meet and surpass its Paris climate targets thanks to aggressive policies. The country receives approximately $70 \%$ of its electricity from coal, but the government wants to integrate $100 \mathrm{GW}$ of solar and $60 \mathrm{GW}$ of wind power into the grid by 2022 , with plans to get almost $60 \%$ of its power from nonfossil sources by 2040 , when the country is anticipated to need more than $1000 \mathrm{GW}$ of power capacity.

"This is a moon-shot initiative to send strong market signals that India is ready to lead on climate," said Kartikeya Singh, a deputy director at the Center for Strategic \& International Studies. "At the same time, new economic reform measures hope to make it easier to do business." Lower interest rates, for instance, have made large-scale utility solar projects more viable and are driving private sector engagement.

However, Singh said, "there is a paradox of plenty.” Industrial demand hasn't increased, putting excess electricity on the grid in some locations. This challenges utilities, which also incur huge losses from a weak grid infrastructure, politicians syphoning power for their constituents, and millions of people not paying for electricity.

The biggest challenge in India now is ensuring affordable power supply to the 50 million households still not on the grid.

The power sector landscape in China has also shifted dramatically in recent years as the nation's economy has slowed its growth. Government policies designed to advance industry, increase energy security, and reduce air pollution have led to the growth of hydropower, solar, and wind outpacing that of coal plants in recent years. Policies such as feed-in tariffs and renewable portfolio standards are boosting renewables, as are sizable investments. In 2016 alone, China spent USD $\$ 87.5$ billion on clean energy deployment, more than $30 \%$ of what the whole world spent.

The scenario is very different in the medium emissions, high-income group of DDPP countries. They already have advanced, low-carbon electricity sectors, which make further deep carbon cuts harder to achieve. 
With Germany, the biggest challenge to decarbonizing is not developing strategies, but implementing them via concrete policies. "One challenge in Europe is the complex regulatory environment and also relatively slow demand growth," said Douglas Arent, executive director of the Joint Institute for Strategic Energy Analysis at the National Renewable Energy Laboratory

The German government wants to cut emissions by $80-95 \%$ below 1990 levels by 2050. The country has committed to phase out nuclear energy, while carbon capture and storage (CCS) lacks public and political support. The main strategy to decarbonize electricity is through renewables, which already produce over a quarter of the country's electricity. Increasing that share requires policies for making the grid flexible to integrate renewables, increasing grid storage, keeping investments stable, and increasing public acceptance. By 2050 , the country aims to get more than $80 \%$ of its electricity from renewables, including biomass, solar, and wind.

In the United States, climate hesitance in the White House could slow down decarbonization efforts, but state and local lawmakers are still pursuing clean power goals, said Arent. Plus, the power landscape is changing due to market forces. Electricity sector emissions are falling because cheap, abundant natural gas is displacing coal for power generation.

Yet several analyses have found that in the long run, abundant natural gas could have little impact on greenhouse gas emissions. Methane leaks from the natural-gas system

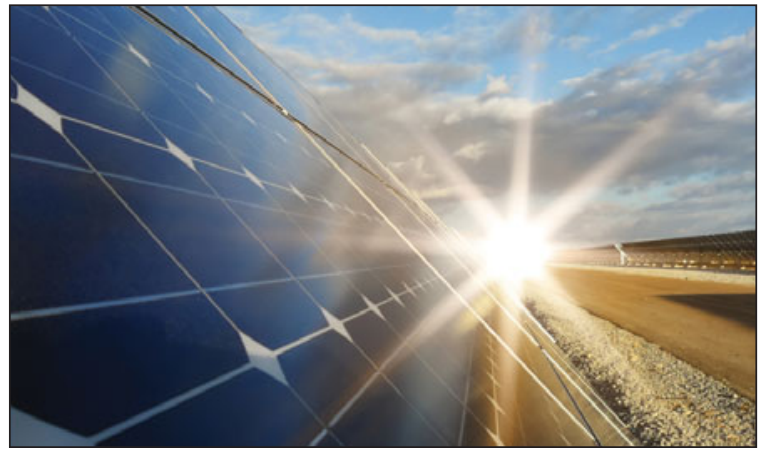

Power plant using renewable solar energy with the sun. Credit: Gencho Petkov.
The right policies will be crucial for deep decarbonization; materials technologies will also set the stage. Photovoltaics (PVs) play out consistently across many nations' low-carbon scenarios. The upside of solar power is that it can be deployed at residential, commercial, and utility scales. Engineering and basic materials advances have already improved solar PV performance and brought down manufacturing costs, especially in China. The cost of solar electricity dropped by half between 2010 and 2014, according to IRENA, with the most competitive utility-scale $\mathrm{PV}$ projects now regularly delivering electricity for just $\$ 0.08$ per $\mathrm{kWh}$.

In China, Germany, and the United States, solar power generation should become cost-competitive with fossil fuels by 2025 , on average. But costs might have to go down further for widespread use, where residential solar (e.g., in rural India and Africa) has to compete with wood-burning stoves and diesel generators.

"What we think of as cheap power is still expensive in some parts of the world," said Benson.

One of the biggest challenges facing solar is grid integration. This will require better integration strategies and utility business models. The upside is that grid solar will drive low-cost energy-storage development. Energy-storage prices are already falling faster than solar PV or wind technologies, according to a recent Nature Energy study. The study by researchers at the University of California and TU Munich in Germany has found that innovation are partly to blame. Some experts believe natural gas should not be the focus of deep decarbonization discussions unless it's coupled with CCS. "In my vision, we can imagine a lot of renewables, some nuclear and in some regions, where there's no good choice other than coal or natural-gas plants, we can use CCS," said Sally Benson, professor of energy engineering at Stanford University and co-director of the University's Precourt Institute for Energy.

However, natural gas is also displacing carbon-free electricity from nuclear sources, which also suffers from large capital expense, waste disposal challenges, and lack of public support. The most recent casualty is the cancellation of two partly finished nuclear reactors in South Carolina.

Renewables, on the other hand, have grown with natural gas in the past five years. Utilities want to have balanced portfolios and make long-term investments based on global energy trends, said Arent. Plus, given the aging coal and grid infrastructure in the United States, economics skew toward new efficient natural-gas plants and distributed energy resources, such as renewables and storage.

There is also a deep cultural shift away from skepticism of new methods and technologies, he added. "Utilities are embracing innovation and the fact that they can manage highly flexible systems and are working on how to operate and be financially viable as the system continues to evolve." $\mathrm{kWh}$ in 2018, down from USD $\$ 10,000 / \mathrm{kWh}$ in the early 1990s.

There is still a need for cheap, durable technologies that can store energy for weeks to account for seasonal solar variations, said Benson. Chemistries such as molten salt batteries could help, as could more investment in flow batteries. "And for the longer term, we could make liquid chemical fuels from solar power as a way of storing solar energy," she added.

Deep decarbonization endeavors will also require private investment. Tesla is now building the world's largest lithiumion storage system in Australia, while German energy company Ewe Gasspeicher $\mathrm{GmbH}$ is building a gigantic redox flow battery facility in underground salt caverns. Such large-scale demonstrations are essential for establishing credibility and reaching economies of scale.

In the long run, to minimize the costs of deep decarbonization, we will need to "ramp up and accelerate all the tools in our toolkit," said Jenkins. Achieving deep decarbonization with renewables alone will be possible, but it will be much more challenging and costly than employing a range of resources. "We need more wind and solar, but also a broader range of lowcarbon technologies in the power sector: biomass, nuclear, and also carbon-capture for natural gas and coal." and investments could bring lithium-ion battery costs to USD $\$ 100$ / 